\title{
A novel test access mechanism for parallel testing of multi-core system
}

\author{
Taewoo Han, Inhyuk Choi, and Sungho Kang ${ }^{\text {a) }}$ \\ Dept. of Electrical and Electronic Eng., University of Yonsei, \\ 134 Sinchon-dong, Seodaemun-gu, Seoul 120-749, Korea \\ a) shkang@yonsei.ac.kr
}

\begin{abstract}
The increased usages of multi-core systems diminish percore complexity and also demand several parallel design and test technologies. This paper introduces a novel test access mechanism (TAM) for parallel testing of multiple identical cores. Instead of typical test response data from the cores, the test output data used in this paper are the majority values extracted from the typical test response from the cores. All the cores can be tested in parallel and test costs (test time, test pins) are exactly the same as for a single core. The experiment results in this paper show the proposed TAM can test multiple cores with minimal test pins and test time and with negligible hardware overhead.
\end{abstract}

Keywords: multi-core, parallel test, TAM, majority

Classification: Integrated circuits

\section{References}

[1] ITRS Test and Test Equipment (2011) 26.

[2] F. Silva, T. McLaurin and T. Waayers: The Core Test Wrapper Handbook: Rationale and Application of IEEE Std. $1500^{T M}$ (Springer-Verlag New York, LLC, 2006).

[3] S. Makar, T. Altinis, N. Patkar and J. Wu: Proceedings IEEE Int. Test Conf. (2007) 9.1.

[4] G. Giles, J. Wang, A. Sehgal, K. J. Balakrishnan and J. Wingfield: Proceedings IEEE Int. Test Conf. (2009) Best Paper.

[5] S. Shamshiri, P. Lisherness, S.-J. Pan and K.-T. Cheng: Proceedings IEEE Int. Test Conf. (2008) 5.3.

[6] OpenSPARCT2: http://www.oracle.com/technetwork/systems/ opensparc/opensparc-t2-page-1446157.html

[7] $90 \mathrm{~nm}$ generic library: http://www.synopsys.com/Comunity/ UniversityProgram

\section{Introduction}

Advances in chip design and test technology have allowed for the integration of a large number of cores into a System-on-Chip (SoC). Multi-core system designs exacerbate power concerns and add test complexity, but also offer exciting Design-for-Test (DFT) opportunities 
[1]. The TAM through which the core can be efficiently accessed and tested is required. Using a general embedded core test methodology such as IEEE 1500 , cores can be wrapped so that there is no interaction with outside data sources, and so that a test for a single core can be generated and applied to each of the instances of that core [2]. Recently, the multi-core designs have evolved to include multiple identical cores and in addition to the benefits of multiprocessing, multiple identical cores can guarantee a highly reliable system by using some cores as redundant cores. Using a broadcast-based TAM to test all of the cores in parallel has been described previously. The AZSCAN architecture tests multiple identical cores in parallel, and the responses are compared with the expected data in the chip [3]. A pipelinebased TAM allows for a great deal of flexibility in test applications, and the pipelining helps to improve test times and to reduce the capture power requirements [4]. They can reduce the test costs of multiple cores to like as single core's, but the necessity of reference cores affects as additional costs.

In this paper, a novel solution to real parallelism - testing multiple cores as a single core is proposed. It uses only the majority value of the multiple cores which is made from the inside of chip. The proposed one can perform a complete core-level diagnosis in case of the multiple cores having faults and can obtain the majority value in one scan shift clock cycle. Therefore, it can use in the both manufacturing test and on-line test. It is related to the delivery of test response data of cores and it can be compatible with the conventional DFT technologies.

\section{TAM for multi-core system}

IEEE 1500 does not prescribe a specific interface connection at the SoC level. Designing SoC TAM architecture is mainly for finding the optimal cost factors such as the test time, test ports, and power dissipation.

Fig. 1 (a) shows a typical TAM architecture for a single-core. The test stimuli patterns for the core are accessed by Test Data Input (TDI) and its test response data are exported by Test Data Output (TDO). The number of pins for TDI, in other words, the test channel bandwidth for TDI is represented by $W$ and it is the same as TDO. Fig. 1 (b) shows a simple diagram of a typical parallel TAM architecture with on-chip comparator [3]. The test response data of multiple identical cores are compared with the expected data in the chip and if any differences appear, 1-bit data ' 1 ' will be recorded in sticky-bit registers (grey blocks in Fig. 1 (b)). After the test processes are finished, only the data in sticky-bit registers are read in the ATE and if they are read as '1's, the related core is considered as a faulty core. Only one TDO which has the same number of pins as a single
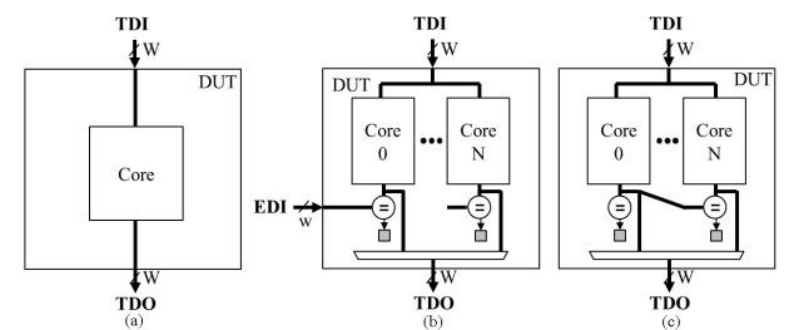

Fig. 1. TAM architectures for signle-core and multi-core systems 
core is used to diagnose a specific core. This parallel TAM can test multiple cores in the same test time as a single core, but needs more test ports for Expected Data Input (EDI). In order to reduce the number of test pins as the same as a single core, a pipelined TAM [4] is proposed which is shown in Fig. 1 (c). The test response data of multiple identical cores are compared on chip with the test response data from a primary core. The test response data of the primary core are compared with the expected data in the ATE and if no faults are detected from the primary core, it can be considered as a non-faulty core and the other cores which have any different test response data from the primary core are faulty cores. This parallel TAM can test multiple cores with the same number of test pins as a single core, but needs additional test time when the primary core is a faulty core.

However, if more than half the number of cores have faults, users practically do not prefer the rest of the cores to salvage it as usable. The multi-core system is derated and has doubt about reliability. Moreover, when the number of redundant cores is increased near to half the number of total cores, the effect of yield improvement which is related to the number of redundant cores decreases [5]. When more than half of the total cores are non-faulty, then the system is salvaged as usable and the proposed TAM can test the cores without a reference of non-faulty cores. If more than half of the total cores are faulty, the proposed TAM can test the multiple cores one by one. However, such chips are usually discarded since it is not cost effective and the performance is too low due to small number of good cores. Therefore, it is a very rare case of testing such chips.

\section{Proposed TAM scheme}

\subsection{Concept of majority-based TAM}

Fig. 2 shows the concept and the principle of the proposed TAM which is called as a majority-based TAM in this paper. The same test patterns are inserted to the cores and Majority Analyzer (MA) module calculates a majority value of the test response data of cores. The majority value is exported to ATE and compared with the test response data of cores in Comparator. In ATE, if the majority value is equal to the expected data, it means that more than half the number of cores do not have faults. Therefore, if the test response data of one core are different from the majority value, it regarded as a faulty core. When the majority value is different from the expected data, it means more than half the number of cores have faults. In this case, one core which has different test response data from the majority value can be a non-faulty core, but this multi-core

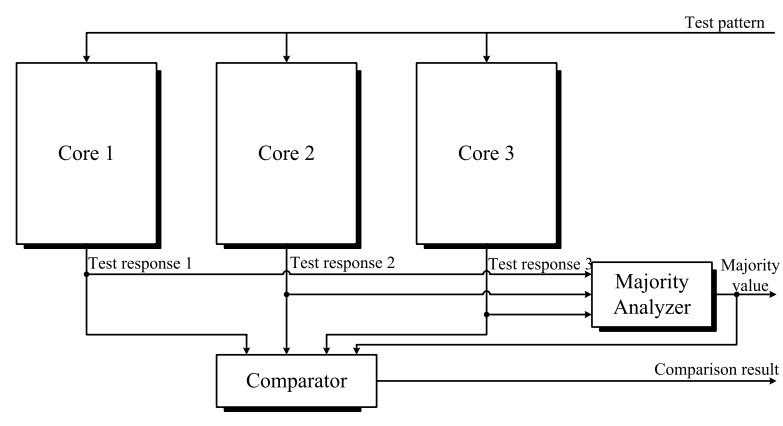

Fig. 2. Concept of majority-based TAM 
chip will be discarded.

\subsection{Architecture of majority-based TAM}

In this paper, a bit-wise MA is designed for simple implementation and efficient operation. Consider the general case in which there are $N$ cores and at least $G$ good cores are required to sell the chip. MA can obtain the majority value by using the number of ' 1 ' in the test response data. If the expected data is ' 1 ', the cores which have ' 0 ' as test response data are faulty cores, and if the number of faulty cores is less than or equal to $N / 2$, this chip has a possibility to be used. In this case, ATE reads faulty cores after the test process is finished and if the number of non-faulty cores is larger than $G$, this chip can be used. On the other hand, if the number of cores which have ' 1 ' as the test response data is larger than $N / 2$ but the expected data is ' 0 ', the cores which have ' 1 ' as test response data are considered as faulty cores and the number of faulty cores turns out to be larger than $N / 2$, this chip cannot be used. Therefore, according to the number of ' 1 's in the test responses of cores and $N / 2$, the majority value is only ' 0 ' or ' 1 ' and it can be simply implemented like as a $N$-to-1 decoder. As a result, the majority-based TAM can select chips which have more than or equal to $G$ good cores. MA is a main module of the proposed TAM and it can decide the majority value by using $N / 2$ as a threshold value.

Fig. 3 shows a majority-based TAM which used the bit-wise MA. It includes a detailed version of MA and the logic of analyzing the majority value can be implemented using combinational logic gates. In MA, one input port can be connected to the output port according to the mux selection signal MA_sel. As a result, the test response data of one specific core are directly connected to the ATE for diagnosis or other purposes.

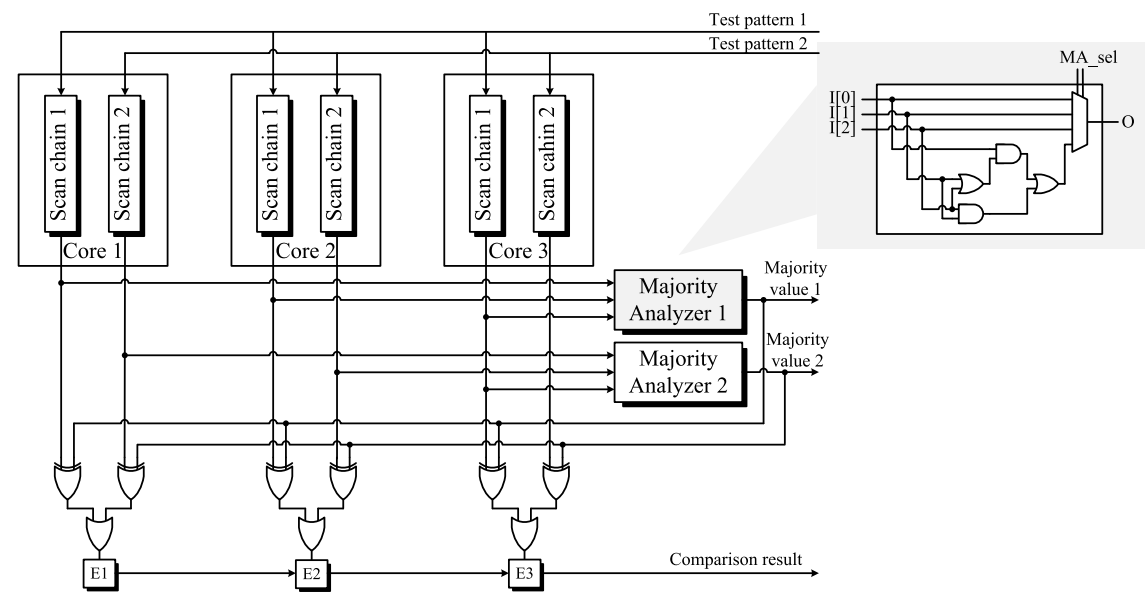

Fig. 3. Architecture of majority-based TAM

The test responses of Scan chain $1 \mathrm{~s}$ are compared with Majority value 1 by XOR gates. If any test response data of scan chains are different from the majority data, that core is regarded as a faulty core and it is recorded at register $\mathrm{E}$ of each core. During the test process, if TDO confirmed to be different from the expected data in the ATE, it means more than half the number of cores have faults and this multi-core system will be discarded. On the other hand, if there are no difference between TDO and the expected data during the whole test process, it means this multi-core system can be salvaged and used. After the whole test process is finished, 
the ATE reads the E registers and the recorded cores are faulty cores and the remained cores are non-faulty cores.

\section{Experimental results}

Several experiments are performed to verify the effectiveness of the proposed parallel TAM. The CPU cores in OpenSPARC T2 [6] are synthesized by Synopsys $90 \mathrm{~nm}$ generic library [7] for analyzing and implementing the proposed TAM in real multi-core system.

Fig. 4 shows the hardware size of the parallel TAMs in the number of NAND gates. The hardware size of proposed TAM is larger than the broadcast-based TAM and smaller than the pipeline-based TAM. Considering the fact that the number of gates of a modern multi-core processor system is much more than million gates, the area overhead of the proposed TAM is negligible.

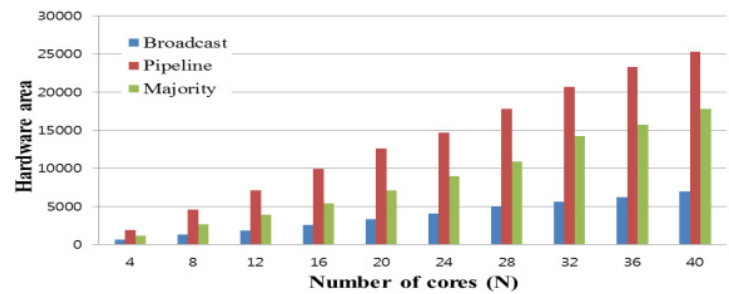

Fig. 4. Comparison of the hardware size of parallel TAMs $(W=32)$

Table I shows the hardware overhead of the proposed TAM to cores and test wrappers. $N$ in the first column represents the number of cores. In Table I, spc (a) is SPARC processor core module and IEEE 1500 (b) indicates the size of IEEE 1500 standard wrapper for the spc module which has 477 ports. Let $S$ be the number of gates $(546,089)$ of a spc and $C$ be the number of gates $(5,151)$ of an IEEE 1500 for the spc, respectively. Majoritybased TAM (c) represents the hardware area of the proposed TAM. TAM overhead in the table indicates the percentage of hardware overhead of the proposed TAM considering both IEEE 1500 standard wrapper for spc module and proposed TAM as the total amount. Total overhead means the percentage of hardware overhead of the proposed TAM divided by the total amount including spc core modules and it is less than $0.1 \%$ in the worst case.

Table I. Hardware overhead of the proposed TAM

\begin{tabular}{|c|c|c|c|c|c|c|c|c|c|c|c|}
\hline \multirow{3}{*}{$\mathrm{N}$} & \multirow{3}{*}{$\frac{\mathrm{spc}}{\text { (a) }}$} & \multirow{3}{*}{$\begin{array}{c}\text { IEEE } \\
1500 \\
\text { (b) }\end{array}$} & \multicolumn{3}{|c|}{ Majority-based TAM } & \multicolumn{3}{|c|}{ TAM overhead } & \multicolumn{3}{|c|}{ Total overhead } \\
\hline & & & \multicolumn{3}{|c|}{ (c) } & \multicolumn{3}{|c|}{$100 * \mathrm{c} /(\mathrm{b}+\mathrm{c})$} & \multicolumn{3}{|c|}{$100 * \mathrm{c} /(\mathrm{a}+\mathrm{b}+\mathrm{c})$} \\
\hline & & & $W=1$ & $W=16$ & $W=32$ & $W=1$ & $W=16$ & $W=32$ & $W=1$ & $W=16$ & $W=32$ \\
\hline 4 & $4 S$ & $4 C$ & 80 & 610 & 1,181 & 0.386 & 2.875 & 5.420 & 0.003 & 0.028 & 0.054 \\
\hline 8 & $8 S$ & $8 C$ & 164 & 1,367 & 2,665 & 0.397 & 3.212 & 6.075 & 0.003 & 0.031 & 0.060 \\
\hline 12 & $12 S$ & $12 C$ & 343 & 2,779 & 5,423 & 0.414 & 3.262 & 6.173 & 0.004 & 0.032 & 0.061 \\
\hline 16 & $16 S$ & $16 C$ & 712 & 6,575 & 14,204 & 0.430 & 3.836 & 7.934 & 0.004 & 0.037 & 0.081 \\
\hline
\end{tabular}

In Table II, $W$ is the number of test pins for a single-core, $T$ is the test time for a single-core, respectively. The proposed TAM needs the same number of test pins as the TAM for a single-core $(2 W=W$ for TDI $+W$ for 
Table II. Comparison of parallel TAMs

\begin{tabular}{c|c|c|c}
\hline & Broadcast[3] & Pipeline[4] & Proposed \\
\hline Test pins & $3 W$ & $2 W$ & $2 W$ \\
\hline Test time (max) & $T$ & $(N-G+1)^{*} T$ & $T$ \\
\hline Hardware overhead (\%) & 0.0288 & 0.1037 & 0.0604 \\
\hline
\end{tabular}

TDO), but the broadcast-based TAM needs more number of test pins for receiving the expected data $(3 W=W$ for TDI $+W$ for TDO $+W$ for EDI). The proposed TAM needs the same test time as the TAM for a single-core, but in the pipeline-based TAM, if $N$ - $G$ tests are conducted in which each of the primary cores fails, then there is one last test to determine whether the remaining $G$ cores are all good. The hardware overhead is the total overhead when $N=8, W=32$ and the results in Table II are derived from the experimental data in Fig. 4 and the calculation method in Table I. All the parallel TAMs need $N$ comparators and in addition, the pipeline-based TAM also needs $N^{*} 2 W$ ( $W$ for TDI and $W$ for TDO $)+4 N(3$ for commands and 1 for match) pipeline registers and the proposed TAM also needs $W$ MAs. Since the area of large number of the pipeline registers is larger than that of MAs, the hardware overhead of the proposed TAM is smaller than the pipeline-based TAM.

\section{Conclusion}

In this paper, we described a new parallel TAM for parallel testing of multicore system. All cores can be tested using the same test pins and the same test time as required for testing a single core. MA module is designed and it uses the majority value for testing multiple identical cores and if the test response data of one core are different from the majority value, the core is regarded as a faulty core. From the view point of the ATE, the multi-core chip with the majority-based TAM can be tested only with the cost for a single-core chip without big modifications. The experimental results show that the proposed TAM has the minimized number of test pins and test time with sufficient operational speed and negligible hardware overhead. It is only related to the delivery of test response data and it can be compatible and improved with the existing DFT technologies. Since the proposed TAM is flexible in design, configuration, and application, it can be used as an attractive and efficient solution to test multiple identical cores.

\section{Acknowledgments}

This research was supported by the Ministry of Science, ICT \& Future Planning (MSIP) of Korea, under the Convergence Information Technology Research Center (C-ITRC) support program (NIPA-2013-H0401-131005) supervised by the National IT Industry Promotion Agency (NIPA). 